\title{
Finite Element Model with Continuous Transverse Shear Stress for Composite Laminates in Cylindrical Bending
}

\author{
K.Y.Sze, Ronggeng Chen* \\ Department of Mechanical Engineering, The University of Hong Kong \\ Pokfulam Road, Hong Kong SAR \\ Y.K.Cheung \\ Department of Civil Engineering, The University of Hong Kong \\ Pokfulam Road, Hong Kong SAR
}

\begin{abstract}
In the double superposition hypothesis, the global inplane displacement, which applies to the whole laminate, is enriched by local displacements which are restricted to each individual ply. To avoid the number of d.o.f.s growing with the number of plies, the transverse shear stress continuity is enforced as usual whereas the inplane displacement continuity is "doubly" constrained for two different groups of the local displacement. Based on the hypothesis, a two-node beam element is attempted. The element has the deflection and its derivative as its nodal d.o.f.s. Despite the fact that interpolated deflection is a cubic function of the longitudinal coordinate, the element yields poor accuracy. The cause is sorted out to be an algebraic constraint in the transverse shear. To overcome the constraint, a heterosis node is added. Remarkable improvement of the element accuracy is noted.
\end{abstract}

keywords : finite element, laminate, double superposition, heterosis, beam

* this research was conducted when Ronggeng Chen was on leave from

Dalian Fisheries College, Dalian 116024, P.R.CHINA

Published in Finite Elements in Analysis and Design 31 (1998) 153-164 


\section{INTRODUCTION}

In the past three decades, considerable interest have been attracted to develop analytical and numerical models for structural/stress analysis of composite laminates. It has been well-known that the classical plate theory, in which the transverse normal and the mid-surface are assumed to be perpendicular before and after deformation, is inadequate [1]. Reissner and Mindlin proposed the first order shear deformation theory as a supplement to the classical theory $[2,3]$. The former allows for a constant transverse shear strain along the thickness direction. Physically, the transverse shear stress should vanish at the top and bottom surfaces of the plate. Hence, a shear correction factor is required to maintain a proper transverse shear stiffness. The factor can be eliminated if higher order shear deformation theory is employed with some of its kinematic d.o.f.s constrained according to the zero shear traction boundary condition [4-6]. Generally speaking, shear deformation theories can determine global responses such as transverse displacement, buckling load and harmonic frequency with acceptable accuracy. However, they fail in predicting the transverse shear stress due to the $C^{1}$-nature of the assumed inplane displacement in the thickness direction that renders the transverse shear strain continuous. With different elastic moduli along and transverse to the fibre, the predicted transverse shear stress becomes discontinuous and, thus, violates the traction reciprocity at the ply- interface.

Layerwise models are the fundamental remedy to the $C^{1}$-limitation of the shear deformation theory. In these model, only $\mathrm{C}^{0}$-continuity of the displacement is maintained at the ply-interfaces [7-17]. To enhance the accuracy of the transverse shear stress in the layerwise model, there are at least three different approaches. In the renowned hybrid/mixed finite element method, an independently assumed $\mathrm{C}^{0}$-continuous transverse shear stress which may also be equilibrating is assumed [10-12]. It is often noted that the inplane stress and its inplane derivatives are satisfactory. The second approach consists of post-processing measures that derive the transverse shear stress from the inplane stress by making use of the stress equilibrium condition [9,17,18]. A shortcoming of the two approaches is that the number of kinematic d.o.f.s will become intractable as the number of plies grows. The third approach enforces the $\mathrm{C}^{0}$-continuity of the transverse shear stress by expressing the condition in terms of the kinematic d.o.f.s and having some of them eliminated. It is possible to limit the number of local kinematic d.o.f.s per ply equal to that of the displacement and transverse shear stress continuity conditions per ply-interface [14-16]. In this light, the number of kinematic d.o.f.s will be unchanged with respect to the number of plies but the restriction on the number of d.o.f.s per ply limits the accuracy of the approach.

Very recently, Li \& Liu has proposed a double superposition hypothesis. The core idea is to enforce the $\mathrm{C}^{0}$-continuity of the inplane displacement separately on two different group of kinematic d.o.f.s in addition to the $\mathrm{C}^{0}$-continuity of the transverse shear stress [16]. The hypothesis 
enriches the polynomial content of the inplane displacement while keeping the net number of d.o.f.s constant and independent to the number of ply. By using the virtual work principle and a set of trial displacement functions which are exact along the inplane direction, Li \& Liu obtained impressive accuracy in the cylindrical bending problems of Pagano [1]. To make the hypothesis more versatile, a pertinent beam finite element model is derived. Numerical examples and their elasticity solutions are presented to illustrate the element accuracy.

\section{THE 1,2-3 DOUBLE SUPERPOSITION HYPOTHESIS}

Details of the hypothesis for plate bending analysis can be found in reference [16]. In this section, the hypothesis has been simplified for beam bending analysis, see Fig.1. The displacement field of the k-th ply in the laminated beam can be written as :

$$
u^{k}(x, z)=u_{G}(x, z)+\bar{u}_{L}^{k}(x, z)+\tilde{u}_{L}^{k}(x, z), w^{k}(x, z)=w_{0}(x)
$$

where

$u_{G}(x, z)=u_{0}(x)+u_{1}(x) z+u_{2}(x) z^{2}+u_{3}(x) z^{3}$ is the global inplane displacement $\bar{u}_{L}^{k}(x, z)=u_{1}^{k}(x) \zeta_{k}+u_{2}^{k}(x) \zeta_{k}^{2}$ is the first group of local (to the k-th ply) inplane displacement $\tilde{u}_{L}^{k}(x, z)=u_{3}^{k}(x) \zeta_{k}^{3}$ is the second group of local (to the k-th ply) inplane displacement $\zeta_{k}=\frac{2 z-z_{k+1}-z_{k}}{z_{k+1}-z_{k}} \in[-1,+1]$ is the transverse parametric coordinate of the $\mathrm{k}$-th ply
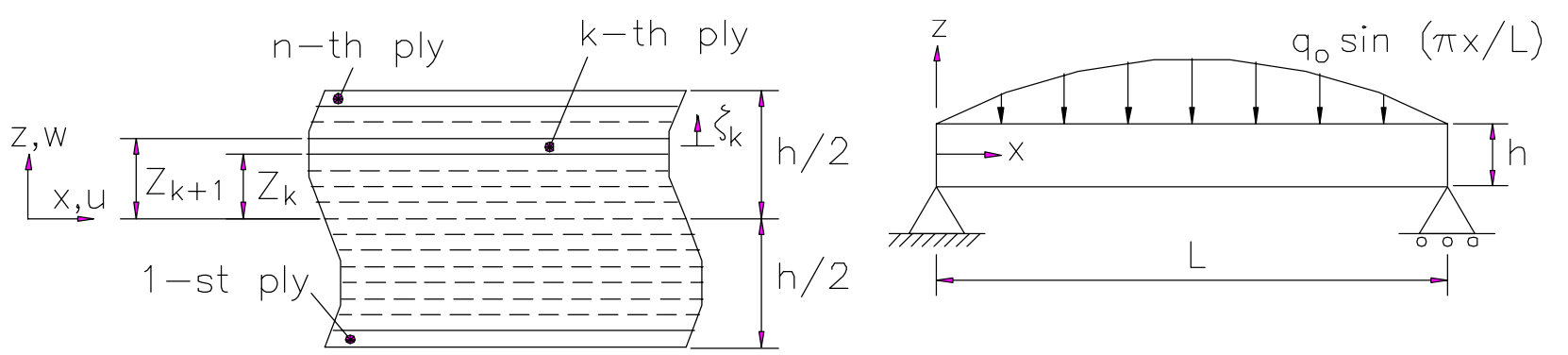

Fig.1. (Left) Schematic diagram for a beam segment. (Right) A simply supported beam loaded by a sinusoidal distributed load.

It can be seen that the k-th ply is bounded by $z=z_{k}$ and $z=z_{k+1}$. Moreover, $\zeta_{k}= \pm 1$ at the two plyinterfaces. This is termed the 1,2-3 hypothesis as the first group of local displacement contains the 1st and 2nd order $\zeta_{k}$-terms whereas the second group of local displacement contains the 3rd order 
$\zeta_{k}$-term. The 1,3-2 and 2,3-1 hypotheses can also be formed. However, we shall restrict ourselves to the 1,2-3 hypothesis as it is most natural.

For a n-ply laminated beam, the number of inplane displacement d.o.f.s is $4+3 n$. To reduce the number of d.o.f.s, the $\mathrm{C}^{0}$-continuity of the transverse shear stress and inplane displacement (i.e. $\left.\bar{u}_{L}^{k}+\tilde{u}_{L}^{k}\right)$ at the ply-interface can be used that result in 2(n-1) constraints. In other words, the number of d.o.f.s would still grow with the number of plies. The double superposition hypothesis suppresses the growth by enforcing the $\mathrm{C}^{0}$-continuity of $\bar{u}_{L}^{k}$ and $\tilde{u}_{L}^{k}$ separately in addition to that of the transverse shear stress. Mathematically, these constraints are :

$$
\bar{u}_{L}^{k-1}\left(x, z_{k}\right)=\bar{u}_{L}^{k}\left(x, z_{k}\right), \tilde{u}_{L}^{k-1}\left(x, z_{k}\right)=\tilde{u}_{L}^{k}\left(x, z_{k}\right), Q^{k-1} \gamma^{k-1}\left(x, z_{k}\right)=Q^{k} \gamma^{k}\left(x, z_{k}\right)
$$

where

$Q^{k}$ is the transverse shear modulus of the k-th ply

$$
\gamma^{k}(x, z)=\frac{\partial w^{k}(x, z)}{\partial x}+\frac{\partial u^{k}(x, z)}{\partial z} \text { is the transverse shear strain in the k-th ply }
$$

By virtue of Eqn.(1), Eqn.(2) can be expressed as :

$$
\begin{aligned}
& u_{2}^{k}=u_{1}^{k}+u_{1}^{k-1}+u_{2}^{k-1}, u_{3}^{k}=-u_{3}^{k-1}, \\
& u_{1}^{k}=-\left(2+\alpha_{k}\right) u_{1}^{k-1}-2\left(1+\alpha_{k}\right) u_{2}^{k-1}-3\left(1+\alpha_{k}\right) u_{3}^{k-1}-\beta_{k}\left(w_{0, x}+u_{1}+2 z_{k} u_{2}+3 z_{k}^{2} u_{3}\right)
\end{aligned}
$$

where

$$
\alpha_{k}=\frac{\left(z_{k+1}-z_{k}\right) Q^{k-1}}{\left(z_{k}-z_{k-1}\right) Q^{k}}, \beta_{k}=\frac{\left(z_{k+1}-z_{k}\right)\left(Q^{k-1}-Q^{k}\right)}{2 Q^{k}}
$$

Eqn.(3) gives the recursive formulae for the local d.o.f.s of the k-th ply in terms of the local d.o.f.s of the (k-1)-th ply. Hence, the displacement described by Eqn.(1) for any ply can be expressed in terms of global and local displacement d.o.f.s of the 1st ply, i.e. $w_{0}(x), u_{0}(x), u_{1}(x), u_{2}(x), u_{3}(x)$, $u_{1}^{1}(x), u_{2}^{1}(x)$ and $u_{3}^{1}(x)$. Moreover, $u_{2}^{1}(x)$ and $u_{3}^{1}(x)$ can be eliminated by the zero shear traction condition at top and bottom surfaces of the laminate, i.e. $Q^{1} \gamma^{1}\left(z=z_{1}\right)=Q^{n} \gamma^{n}\left(z=z_{n+1}\right)=0$. Finally, the displacement field of the k-th layer can be expressed as :

$$
\begin{aligned}
& u^{k}(x, z)=u_{o}(x)+f_{1}^{k}(z) u_{1}^{1}(x)+f_{2}^{k}(z) u_{1}(x)+f_{3}^{k}(z) u_{2}(x)+f_{4}^{k}(z) u_{3}(x)+f_{5}^{k}(z) w_{o, x}(x) \\
& w^{k}(x, z)=w_{o}(x)
\end{aligned}
$$


where

$$
\begin{aligned}
& f_{1}^{k}(z)=R_{5}^{k} \zeta_{k}+S_{5}^{k} \zeta_{k}^{2}+T_{5}^{k} \zeta_{k}^{3}, f_{2}^{k}(z)=R_{1}^{k} \zeta_{k}+S_{1}^{k} \zeta_{k}^{2}+T_{1}^{k} \zeta_{k}^{3} \\
& f_{3}^{k}(z)=z+R_{2}^{k} \zeta_{k}+S_{2}^{k} \zeta_{k}^{2}+T_{2}^{k} \zeta_{k}^{3}, f_{4}^{k}(z)=z^{2}+R_{3}^{k} \zeta_{k}+S_{3}^{k} \zeta_{k}^{2}+T_{3}^{k} \zeta_{k}^{3} \\
& f_{5}^{k}(z)=z^{3}+R_{4}^{k} \zeta_{k}+S_{4}^{k} \zeta_{k}^{2}+T_{4}^{k} \zeta_{k}^{3}
\end{aligned}
$$

To compute $R_{i}^{k}$ 's $S_{i}^{k}$ 's and $T_{i}^{k}$ 's, we first define :

$$
\begin{aligned}
& F_{1}^{1}=1, F_{2}^{1}=F_{3}^{1}=F_{4}^{1}=F_{5}^{1}=F_{6}^{1}=0, G_{2}^{1}=1, G_{1}^{1}=G_{3}^{1}=G_{4}^{1}=G_{5}^{1}=G_{6}^{1}=0, \\
& H_{1}^{1}=-\frac{1}{3}, H_{2}^{1}=\frac{2}{3}, H_{3}^{1}=-\frac{\left(z_{2}-z_{1}\right)}{6}, H_{4}^{1}=-\frac{z_{1}\left(z_{2}-z_{1}\right)}{3}, H_{5}^{1}=-\frac{z_{1}^{2}\left(z_{2}-z_{1}\right)}{2}, H_{6}^{1}=-\frac{\left(z_{2}-z_{1}\right)}{6}
\end{aligned}
$$

For $k>1, F_{i}^{k}, G_{i}^{k}$ and $H_{i}^{k}$ are calculated from the following recursive relations :

$$
\begin{aligned}
& H_{1}^{k}=-H_{1}^{k-1}, H_{2}^{k}=-H_{2}^{k-1}, H_{3}^{k}=-H_{3}^{k-1}, H_{4}^{k}=-H_{4}^{k-1}, H_{5}^{k}=-H_{5}^{k-1}, H_{6}^{k}=-H_{6}^{k-1}, \\
& F_{1}^{k}=-\left(2+\alpha_{k}\right) F_{1}^{k-1}-2\left(1+\alpha_{k}\right) G_{1}^{k-1}-3\left(1+\alpha_{k}\right) H_{1}^{k-1}, \\
& F_{2}^{k}=-\left(2+\alpha_{k}\right) F_{2}^{k-1}-2\left(1+\alpha_{k}\right) G_{2}^{k-1}-3\left(1+\alpha_{k}\right) H_{2}^{k-1}, \\
& F_{3}^{k}=-\left(2+\alpha_{k}\right) F_{3}^{k-1}-2\left(1+\alpha_{k}\right) G_{3}^{k-1}-3\left(1+\alpha_{k}\right) H_{3}^{k-1}-\beta_{k}, \\
& F_{4}^{k}=-\left(2+\alpha_{k}\right) F_{4}^{k-1}-2\left(1+\alpha_{k}\right) G_{4}^{k-1}-3\left(1+\alpha_{k}\right) H_{4}^{k-1}-2 \beta_{k} z_{k}, \\
& F_{5}^{k}=-\left(2+\alpha_{k}\right) F_{5}^{k-1}-2\left(1+\alpha_{k}\right) G_{5}^{k-1}-3\left(1+\alpha_{k}\right) H_{5}^{k-1}-3 \beta_{k} z_{k}^{2}, \\
& F_{6}^{k}=-\left(2+\alpha_{k}\right) F_{6}^{k-1}-2\left(1+\alpha_{k}\right) G_{6}^{k-1}-3\left(1+\alpha_{k}\right) H_{6}^{k-1}-\beta_{k}, \\
& G_{1}^{k}=F_{1}^{k-1}+F_{1}^{k}+G_{1}^{k-1}, G_{2}^{k}=F_{2}^{k-1}+F_{2}^{k}+G_{2}^{k-1}, G_{3}^{k}=F_{3}^{k-1}+F_{3}^{k}+G_{3}^{k-1}, \\
& G_{4}^{k}=F_{4}^{k-1}+F_{4}^{k}+G_{4}^{k-1}, G_{5}^{k}=F_{5}^{k-1}+F_{5}^{k}+G_{5}^{k-1}, G_{6}^{k}=F_{6}^{k-1}+F_{6}^{k}+G_{6}^{k-1}
\end{aligned}
$$

Finally, $R_{i}^{k}$ 's $S_{i}^{k}$ 's and $T_{i}^{k}$ 's required by $f_{i}^{i}(z)$ 's are computed as :

$$
\begin{array}{lll}
R_{1}^{k}=F_{1}^{k}+A_{1} F_{2}^{k}, & S_{1}^{k}=G_{1}^{k}+A_{1} G_{2}^{k}, & T_{1}^{k}=H_{1}^{k}+A_{1} H_{2}^{k}, \\
R_{2}^{k}=F_{3}^{k}+B_{1} F_{2}^{k}, & S_{2}^{k}=G_{3}^{k}+B_{1} G_{2}^{k}, & T_{2}^{k}=H_{3}^{k}+B_{1} H_{2}^{k}, \\
R_{3}^{k}=F_{4}^{k}+C_{1} F_{2}^{k}, & S_{3}^{k}=G_{4}^{k}+C_{1} G_{2}^{k}, & T_{3}^{k}=H_{4}^{k}+C_{1} H_{2}^{k}, \\
R_{4}^{k}=F_{5}^{k}+D_{1} F_{2}^{k}, & S_{4}^{k}=G_{5}^{k}+D_{1} G_{2}^{k}, & T_{4}^{k}=H_{5}^{k}+D_{1} H_{2}^{k}, \\
R_{5}^{k}=F_{6}^{k}+E_{1} F_{2}^{k}, & S_{5}^{k}=G_{6}^{k}+E_{1} G_{2}^{k}, & T_{5}^{k}=H_{6}^{k}+E_{1} H_{2}^{k}
\end{array}
$$


in which

$$
A_{1}=-\frac{\Delta_{1}^{1}}{\Delta_{1}^{2}}, B_{1}=-\frac{1+\Delta_{1}^{3}}{\Delta_{1}^{2}}, C_{1}=-\frac{2 z_{n+1}+\Delta_{1}^{4}}{\Delta_{1}^{2}}, D_{1}=-\frac{3 z_{n+1}^{2}+\Delta_{1}^{5}}{\Delta_{1}^{2}}, E_{1}=-\frac{1+\Delta_{1}^{6}}{\Delta_{1}^{2}}
$$

and

$$
\Delta_{1}^{i}=2\left(F_{i}^{n}+2 G_{i}^{n}+3 H_{i}^{n}\right) /\left(z_{n+1}-z_{n}\right)
$$

Li \& Liu has tested their hypothesis with the simply supported laminated beam problem of Pagano [1], see Fig.1. The beam is in plane strain condition and plied at $0^{\circ}$ and $90^{\circ}$ with respect to the $\mathrm{x}$ axis. The material constants are :

$$
E_{L}=25 \times 10^{6}, E_{T}=10^{6}, G_{L T}=0.5 \times 10^{6}, G_{T T}=25 \times 10^{6}, v_{L T}=v_{T T}=0.25
$$

where " $L$ " and " $T$ " denote the longitudinal and transverse directions with respect to the running fibre. In this problem, the closed-form solutions take the following forms :

$$
u=g_{1}(z) \cos \frac{\pi x}{L}, w=g_{2}(z) \sin \frac{\pi x}{L}
$$

from which the stress components will assume the following forms :

$$
\sigma_{x}=g_{3}(z) \sin \frac{\pi x}{L}, \sigma_{z}=g_{4}(z) \sin \frac{\pi x}{L}, \tau_{y z}=g_{5}(z) \cos \frac{\pi x}{L}
$$

where $g_{i}(z)$ 's are functions of $z$. With the following global trial functions :

$$
\begin{aligned}
& u_{0}=U_{0} \cos \frac{\pi x}{L}, u_{1}^{1}=U_{1}^{1} \cos \frac{\pi x}{L}, u_{1}=U_{1} \cos \frac{\pi x}{L}, \\
& u_{2}=U_{2} \cos \frac{\pi x}{L}, u_{3}=U_{3} \cos \frac{\pi x}{L}, w_{0}=W_{0} \cos \frac{\pi x}{L}
\end{aligned}
$$

which have the same transcendental content as the analytical solutions, Li \& Liu solved the unknown coefficients $U_{0}, U_{1}^{1}, U_{1}, U_{2}, U_{3}$ and $W_{0}$ by the double superposition hypothesis and the virtual work principle. The computed solutions are in good agreement with their analytical counterparts [16]. 
Being impressed by the accurate results in the above simply supported beam problem, the present paper will derive a beam finite element model using the double superposition hypothesis.

\section{TWO-NODE BEAM ELEMENT}

Judging from the kinematic relations given in Eqn.(4), the simplest element appears to be the two-node elements with seven d.o.f.s $\left(u_{o}, w_{o}, w_{o, x}, u_{1}^{1}, u_{1}, u_{2}\right.$ and $\left.u_{3}\right)$ per node, see Fig.2. The interpolated displacements and x-coordinate inside the element "e" can be obtained in the standard way :

$$
\begin{aligned}
& w_{o e}(\xi)=\frac{1}{4}(2+\xi)(1-\xi)^{2} w_{o(1)}+\frac{1}{4}(2-\xi)(1+\xi)^{2} w_{o(2)}+\frac{l_{e}}{8}(1-\xi)^{2}(1+\xi) w_{o, x(1)}-\frac{l_{e}}{8}(1-\xi)(1+\xi)^{2} w_{o, x(2)}, \\
& u_{e}^{k}(\xi, z)=\sum_{i=1}^{2} N_{i}(\xi)\left(u_{o(i)}+f_{1}^{k}(z) u_{1(i)}^{1}+f_{2}^{k}(z) u_{1(i)}+f_{3}^{k}(z) u_{2(i)}+f_{4}^{k}(z) u_{3(i)}\right)+f_{5}^{k}(z) w_{o e, x} \\
& x_{e}(\xi)=N_{1}(\xi) x_{(1)}+N_{2}(\xi) x_{(2)}
\end{aligned}
$$

where

$N_{1}(\xi)=(1-\xi) / 2, N_{2}(\xi)=(1+\xi) / 2, l_{e}=x_{(2)}-x_{(1)}$ is the element length

the subscript "(i)" denotes the nodal counterpart of the related variable
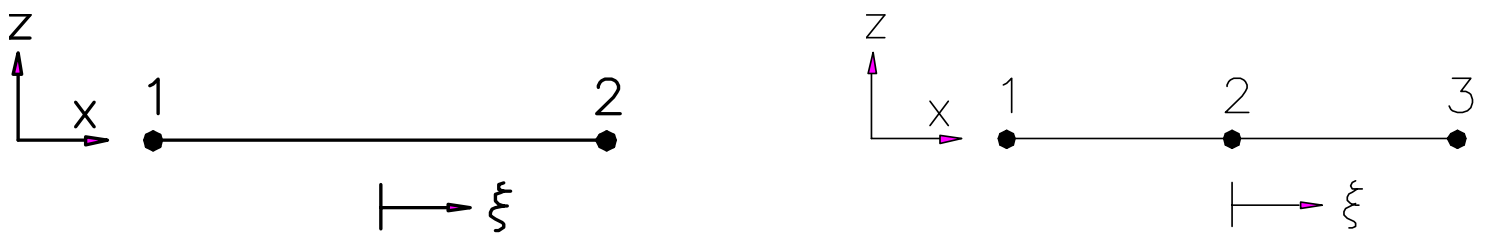

Fig.2. (Left) Two-node beam element. (Right) Three-node heterosis beam element.

With the displacement interpolation known, the element equation can be derived with the virtual work principle. 

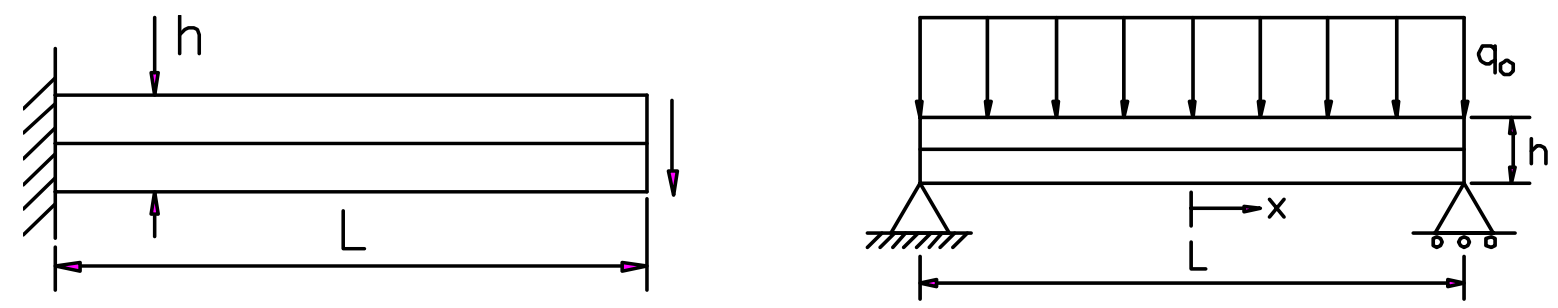

Fig.3. (Left) A cantilever subjected to end shear force. (Right) A simply support beam subjected to uniformly distributed force. Both beams are made of homogeneous isotropic materials with zero Poisson's ratio and modelled by two-ply elements.

Cantilever subjected to End Shear Force: A cantilever made of a homogeneous isotropic material is first examined, see Fig.3. For simplicity, the Poisson's ratio is taken to be zero. The cantilever is modelled by the element using two plies. At the clamped end, all nodal d.o.f.s are restrained. As both the element and exact transverse displacements are cubic in $\xi$ [19], accurate predicted deflection is expected even with only one element. Nevertheless, Fig.4 shows that the predicted end deflection is far from expectation.
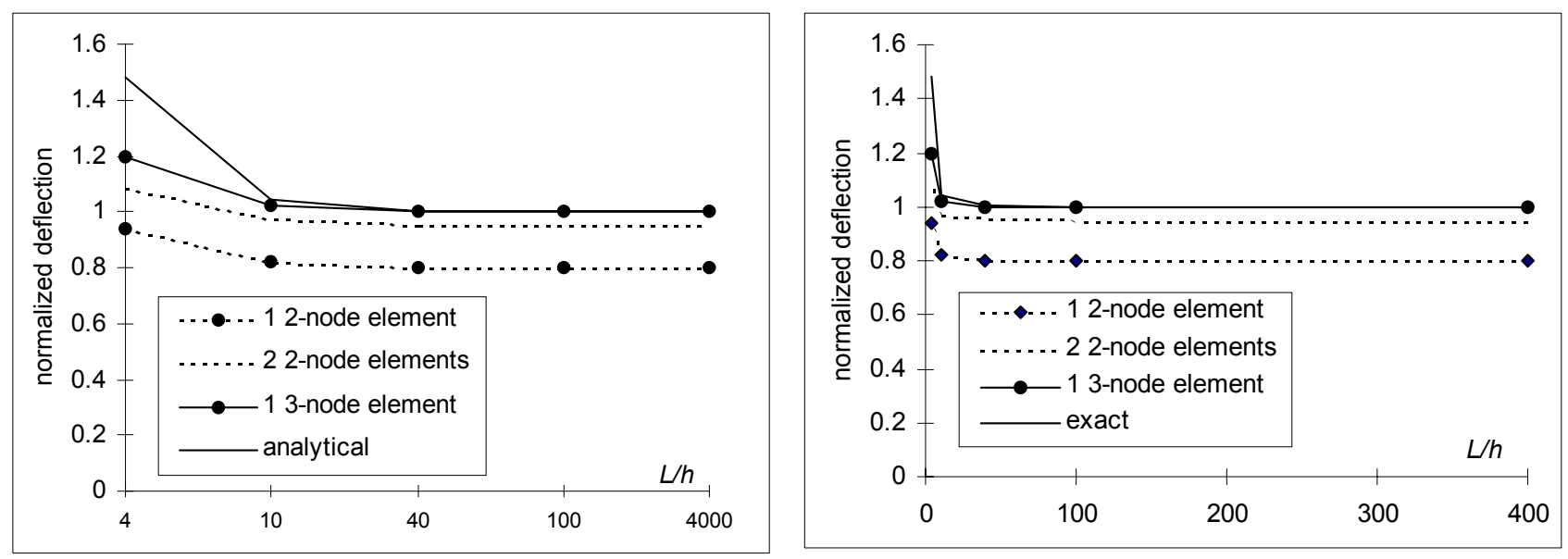

Fig.4. (Left) End deflections for the cantilever problem and (right) mid-span deflections for the simply-supported beam problem, see Fig.3. The deflections have been normalized by the thin beam solution.

Simply-Supported Beam subjected to Uniformly Distributed Loading : The element is also tested by a simply-supported beam made of homogeneous isotropic material with zero Poisson's ratio. Again, the beam is divided into two identical plies. The beam is subjected to uniformly distributed loading $q_{o}$ (force per unit area), see Fig.3. Owing to symmetry, only half of the beam is modelled. Fig.4 shows the normalized mid-span deflections. With two elements, the yielded accuracy is around $95 \%$ at $L / h=10$. However, the transverse shear stress at the two element centres is disappointing as seen in Fig.5. It is noteworthy that the stress is not even symmetric. 

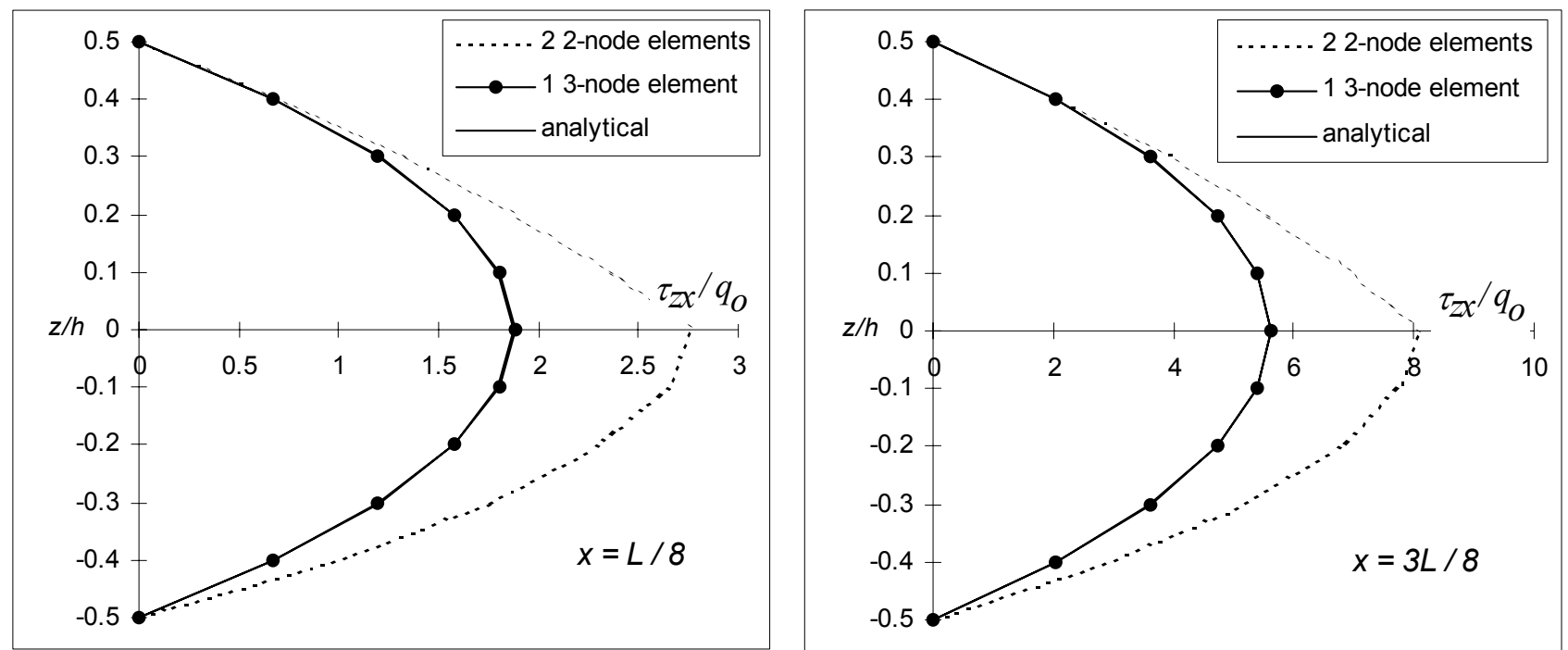

Fig.5. Transverse variation of the normalized transverse shear stress, $\tau_{z x} / q_{o}$, for isotropic beams at (left) $x=L / 8$ and (right) $x=3 L / 8$.

The cause for the unexpected poor accuracy of the element is found to be the coefficient of $\xi^{2}$ in the transverse shear strain or the inplane derivative of the transverse deflection. By recalling Eqn.(10), the coefficient is :

$$
\frac{3}{2 l_{e}}\left(1+\frac{d f_{5}^{k}(z)}{d z}\right)\left\{w_{o(1)}-w_{o(2)}+\frac{l_{e}}{4}\left(w_{o, x(1)}+w_{o, x(2)}\right)\right\}
$$

This presents a severe shear constraint in the sense that it involves very few nodal d.o.f.s. In the two considered problems, the effect of the constraint is obvious even when the element aspect ratio $l_{e} / h$ equals 5 .

\section{THREE-NODE HETEROSIS BEAM ELEMENT}

As mentioned in the last section, the severity of the constraint is that it involves too few nodal d.o.f.s. This is due to the different interpolation orders of $\xi$ employed in the inplane and transverse displacements which are linear and cubic, respectively. To overcome the problem, one can reduce the difference from two to one. In this light, a mid-length heterosis node equipped with only inplane displacement d.o.f.s is introduced. The element displacements become : 


$$
\begin{aligned}
& w_{o e}(\xi)=\frac{1}{4}(2+\xi)(1-\xi)^{2} w_{o(1)}+\frac{1}{4}(2-\xi)(1+\xi)^{2} w_{o(2)}+\frac{l_{e}}{8}(1-\xi)^{2}(1+\xi) w_{o, x(1)}-\frac{l_{e}}{8}(1-\xi)(1+\xi)^{2} w_{o, x(2)}, \\
& u_{e}^{k}(\xi, z)=\sum_{i=1}^{3} \widetilde{N}_{i}(\xi)\left(u_{o(i)}+f_{1}^{k}(z) u_{1(i)}^{1}+f_{2}^{k}(z) u_{1(i)}+f_{3}^{k}(z) u_{2(i)}+f_{4}^{k}(z) u_{3(i)}\right)+f_{5}^{k}(z) w_{o e, x}
\end{aligned}
$$

where

$$
\tilde{N}_{1}(\xi)=\xi(\xi-1) / 2, \tilde{N}_{2}(\xi)=1-\xi^{2}, \tilde{N}_{3}(\xi)=\xi(\xi+1) / 2
$$

The present three-node element has nineteen nodal d.o.f.s but the five d.o.f.s belonging to the heterosis node can be condensed prior to element assembly. With the enhanced inplane displacement, the coefficient of $\xi^{2}$ in the transverse shear strain involve all element nodal d.o.f.s. The accuracy of the new element is far more superior to that of the two-node element both in deflection and shear stress as seen in Fig.4 and Fig.5. Both problems are re-examined with only one three-node element using two plies.

\section{FURTHER NUMERICAL TESTS}

The three-node element is then tested by Pagano's laminated beam problem [1], see the description in Section 2 and Fig. 1 for details. Owing to symmetry, only half of the beam is modelled. The following normalized quantities are used in the subsequent figures :

$$
\begin{aligned}
& \text { normalized transverse shear at beam tip : } \bar{\tau}_{z x}=\frac{\tau_{z x}(x=0)}{q_{0}} \\
& \text { normalized inplane displacement at beam tip : } \bar{u}=\frac{E_{T} u(x=0)}{h q_{0}} \\
& \text { normalized deflection at mid-span : } \bar{w}=\frac{100 E_{T} h^{3} w_{o}(x=L / 2)}{q_{o} L^{4}}
\end{aligned}
$$

As the transverse variation of the transverse shear stress is most drastic for small aspect ratios $(L / h)$, most of the examples employ an aspect ratio equal to four.

(0/90/0) Symmetric Laminate : Fig.6 (left) indicates that excellent central deflection is obtained even with one element and the result is not susceptible to the aspect ratio. On the other hand, accurate inplane displacement at beam tip is achieved with two elements as portrayed in Fig.6 
(right). Fig.7 shows the transverse shear stress for $L / h=4$ (left) and $L / h=10$ (right). With four elements, the predicted stress overlap with the exact solution [1].
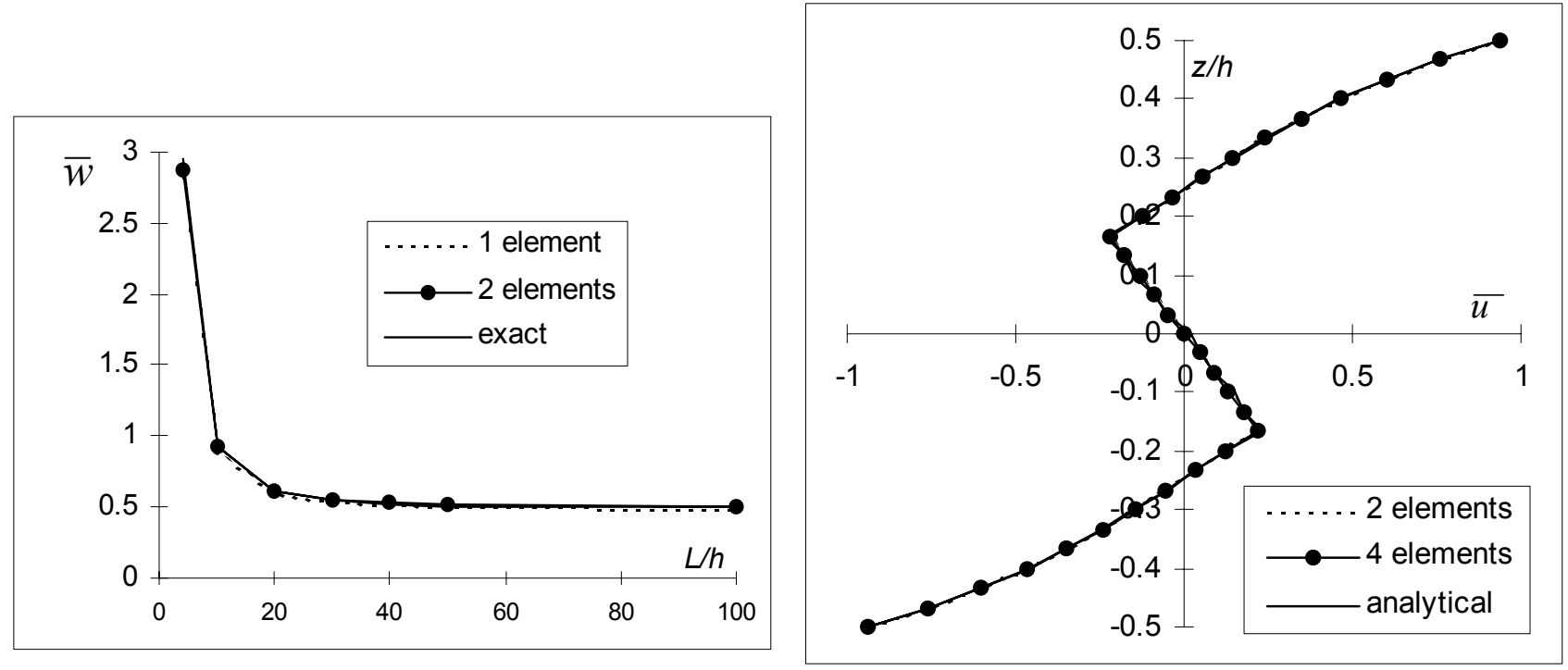

Fig.6. (Left) Normalized deflection, $\bar{w}$, versus aspect ratio and (right) transverse variation of normalized inplane displacement, $\bar{u}$, for $L / h=4(0 / 90 / 0)$ laminate.
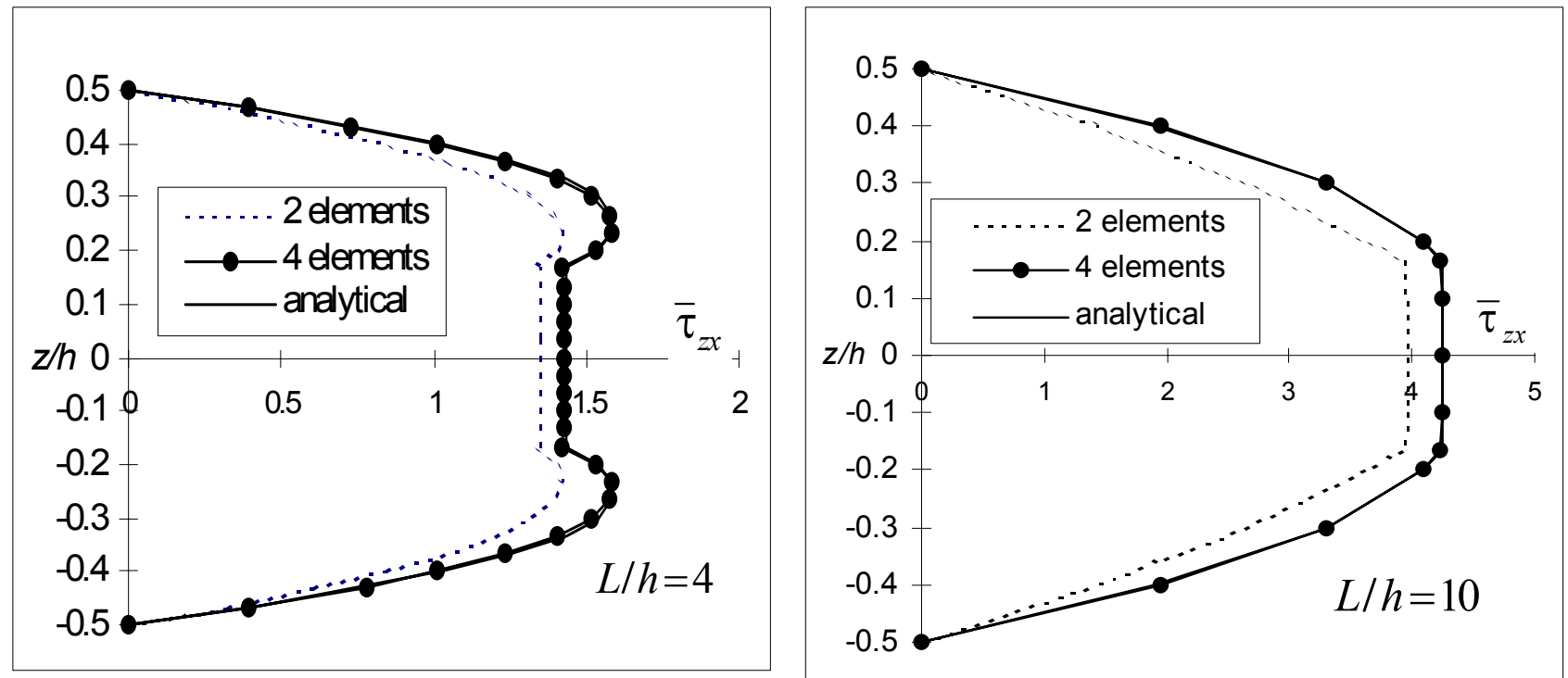

Fig.7. Transverse variations of normalized transverse shear stress, $\bar{\tau}_{z x}$, for (left) $L / h=4$ and (right) $L / h=10$ for $(0 / 90 / 0)$ symmetric laminate.

(0/90/0/90/0/90/0) Symmetric Laminate: Fig.8 shows the transverse variation of the inplane displacement and transverse shear stress for $L / h=4$. The displacement converges with two elements whereas the stress converges with eight elements. While the converged displacement overlaps with its analytical counterpart, the converged stress is in good agreement with its analytical counterpart [1]. 

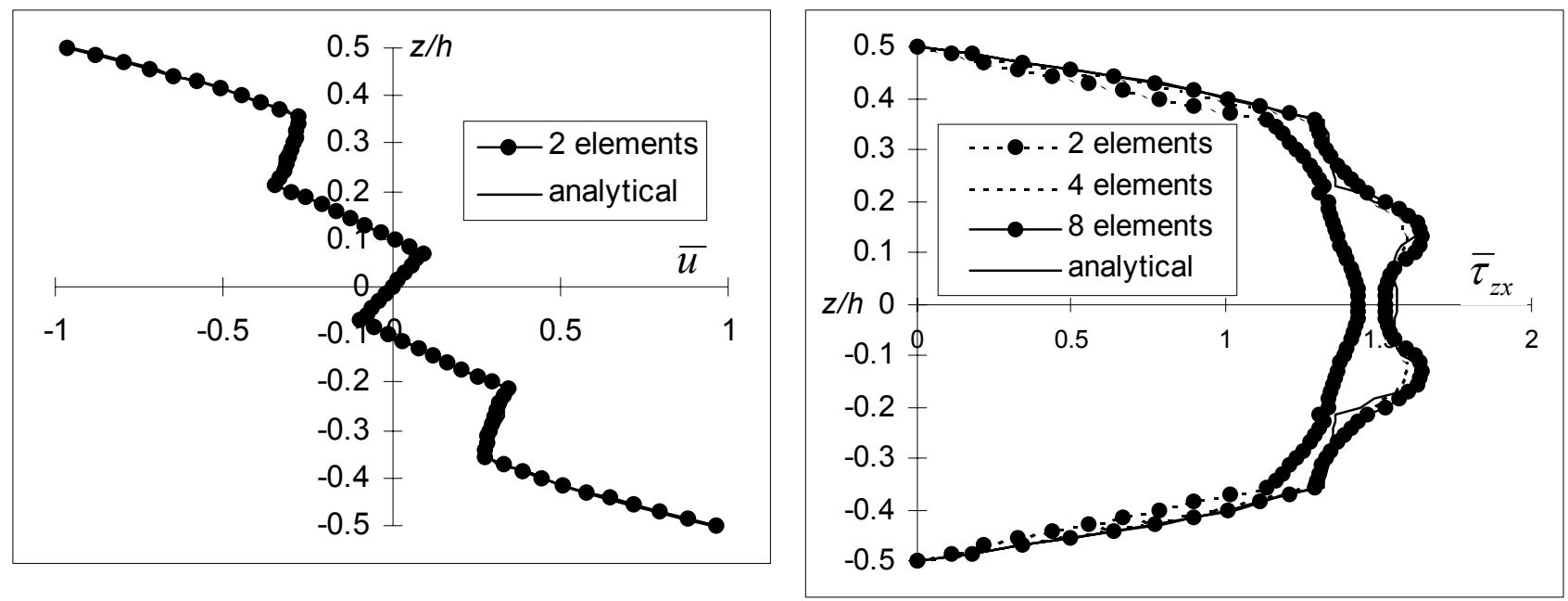

Fig.8. Transverse variation of (left) normalized inplane displacement, $\bar{u}$, and normalized transverse shear stress, $\bar{\tau}_{z x}$, for $(0 / 90 / 0 / 90 / 0 / 90 / 0)$ symmetric laminate, $L / h=4$.

(0/90) Unsymmetric Cross-Ply Laminate : Fig.9 (left) shows the transverse variation of the transverse shear stress for $L / h=4$. By using four elements, the stress converges and is in excellent agreement with the analytical solution.
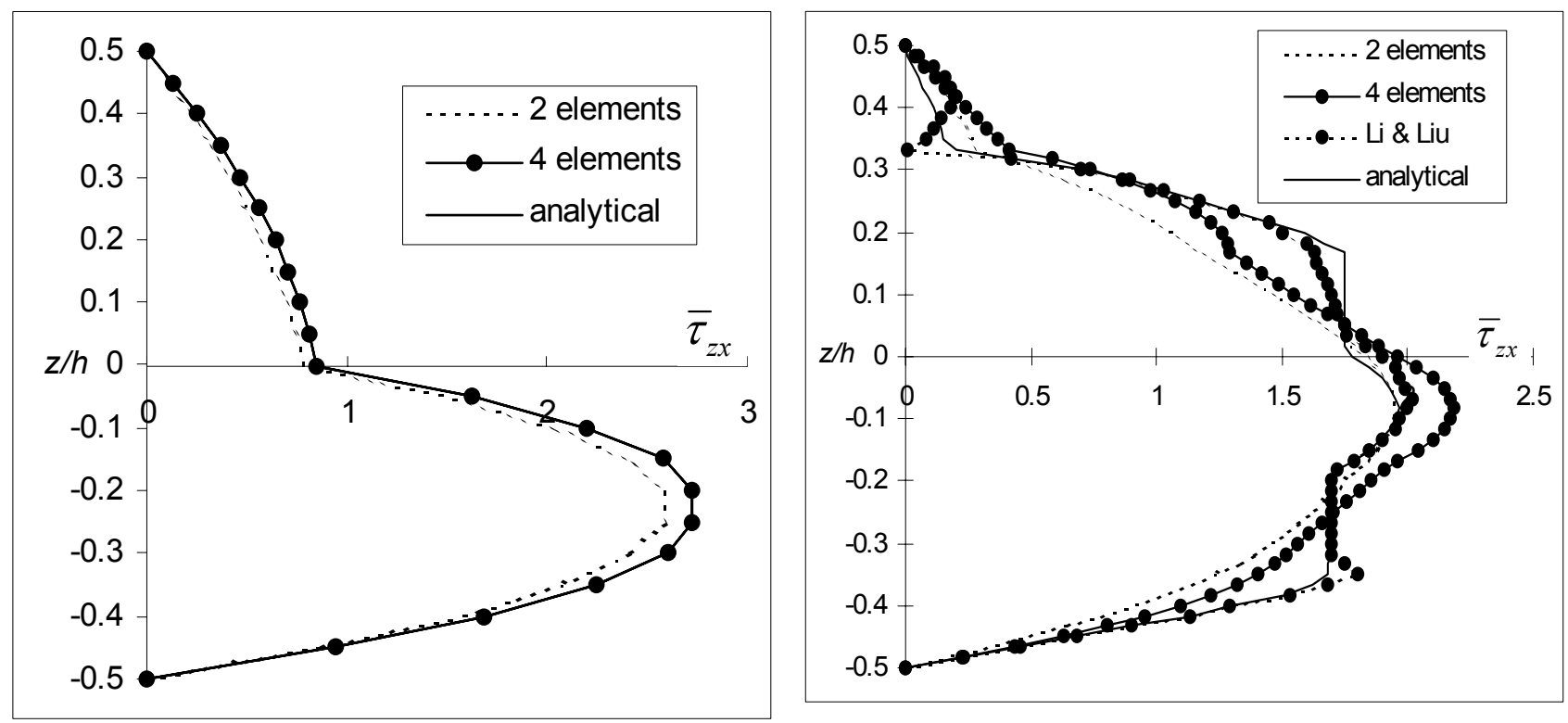

Fig.9. Transverse variations of normalized transverse shear stress, $\bar{\tau}_{z x}$, for (left) $(0 / 90)$ unsymmetric laminate and (right) (0/90/0/90/0/90) unsymmetric laminate, $L / h=4$

(0/90/0/90/0/90) Unsymmetric Cross-Ply Laminate : Fig.9 (right) shows the transverse variation of the transverse shear stress for $L / h=4$. The finite element prediction converges with four elements and does not get closer to the analytical solution with more elements. It can be seen that the present result is not as close to the analytical solution as compared to that of Li \& Liu [16]. This is probably due to the exact transcendental nature of the trial displacement assumed by Li \& Liu, see Eqn.(9). 
Nevertheless, the present result is more smooth as seen at the ply-interfaces of the first and last plies.

\section{CLOSURE}

A three-node heterosis beam element is developed for cylindrical bending analysis of composite laminates by adopting the double superposition hypothesis of Li \& Liu [16]. The heterosis node is introduced to balance the interpolation order of the inplane and transverse displacement d.o.f.s such that the shear constraint observed in the two-node element can be overcome. As revealed in the numerical examples, the element yields excellent and good accuracy in the displacement and stress predictions, respectively. Development of a plate bending element for general laminate analysis is being undertaken.

Acknowledgment - The financial support of the University of Hong Kong in form of a CRCG grant is gratefully acknowledged. 


\section{REFERENCES}

1. N.J.Pagano, "Exact solutions for composite laminates in cylindrical bending", J.Composite Mater., 3, 398-411 (1969)

2. E.Reissner, "The effect of transverse shear deformation on the bending of elastic plates", J.Appl.Mech. - Trans.ASME, 12, A69-A77 (1945)

3. R.D.Mindlin, "Influence of rotatory inertia and shear deformation on flexural motions of isotropic elastic plates", J.Appl.Mech.-Trans.ASME, 18, 31-38 (1951)

4. K.H.Lo, R.M.Christensen, E.M.Wu, "A high-order theory of plate deformation - Part 1 : homogeneous plates", J.Appl.Mech.-Trans.ASME, 44, 663-668 (1977)

5. J.N.Reddy, "A simple higher-order theory for laminated composite plates", J.Appl.Mech. Trans.ASME, 51, 745-752 (1984)

6. A.K.Noor, W.S.Burton, "Assessment of shear deformation theories for multilayered composite plates", Applied Mechanics Review., 42, 1-12 (1989)

7. S.Srinivas, "A refined analysis of composite laminates", J.Sound \& Vibration, 30, 495-507 (1973)

8. E.J.Barbero, J.N.Reddy, J.L.Teply, "A plate bending element based on a generalized laminate plate theory", Inter.J.Numer.Methods Engrg., 28, 2275-2292 (1989)

9. E.J.Barbero, J.N.Reddy, "An accurate determination of stresses in thick laminates using a generalized plate theory", Inter.J.Numer.Methods Engrg., 29, 1-14 (1990)

10. S.T.Mau, P.Tong and T.H.H.Pian, "Finite element solution for laminated thick plates", J.Composite Materials, 6, 304-311 (1972)

11. H.-S.Jing, M.-L.Liao, "Partial hybrid stress element for the analysis of thick laminated composite plates", Inter.J.Numer.Methods Engrg., 28, 2813-2827 (1989)

12. J.Han, S.V.Hoa, "A three-dimensional multilayer composite finite element for stress analysis of composite laminates", Inter.J.Numer.Methods Eng., 36, 3903-3914 (1993)

13. X.Li, D.Liu, "A laminate theory based on global-local superposition", Comm.Numer.Methods. Engrg., 11, 633-641 (1995)

14. A.S.Mawenya, J.D.Davies, "Finite element bending analysis of multilayer plates", Inter.J. Numer.Methods Eng., 8, 215-225 (1974)

15. M.D.Sciuva, "A third-order triangular multilayered plate finite element with continuous interlaminar stresses”, Inter.J.Numer.Methods Eng., 38, 1-26 (1995)

16. X.Li, D.Liu, "Generalized laminate theories based on double suerpothesis", Inter.J.Numer. Methods Eng., 40, 1197-1212 (1997)

17. R.A.Chaudhri, "An equilibrium method for prediction of transverse shear stresses in an thick laminated plate", Computers \& Structures, 23, 139-146 (1986)

18. J.V.Kouri, S.N.Atluri, "Analytical modelling of laminated composites", Composites Science \& Technology, 46, 335-344 (1993)

19. S.P.Timoshenko, J.N.Goodier, Theory of Elasticity, 3rd Edn., McGraw-Hill, New York, 1982 